\title{
The microwave spectrum and structure of the neon-phosphorus trifluoride complex
}

\author{
Kurt W. Hillig II, Marabeth S. LaBarge ${ }^{1}$, Amine Taleb-Bendiab and Robert L. Kuczkowski \\ Department of Chemistry, University of Michigan, Ann Arbor, MI 48109-1055, USA
}

Received 9 April 1990; in final form 22 May 1990

\begin{abstract}
Symmetric top spectra were observed for the ${ }^{20} \mathrm{Ne} \cdot \mathrm{PF}_{3}$ and ${ }^{22} \mathrm{Ne} \cdot \mathrm{PF}_{3}$ van der Waals dimers using a Fourier-transform microwave spectrometer. The center-of-mass distance between $\mathrm{Ne}$ and $\mathrm{PF}_{3}$ is $3.373(3) \AA$. The experimental data, in conjunction with the van der Waals radii of the atoms and with ab initio calculations, are consistent with the neon atom on the symmetry axis over the $\mathrm{F}_{3}$ face of the $\mathrm{PF}_{3}$. Normal mode analysis of the van der Waals vibrations based on the centrifugal distortion constants yields force constants $f_{R}=0.00647 \mathrm{mdyn} \AA^{-1}$ and $f_{\theta}=0.00464$ mdyn $\AA$ for the ${ }^{20} \mathrm{Ne} \cdot \mathrm{PF}_{3}$ isotopic species.
\end{abstract}

The $\mathrm{Ar} \cdot \mathrm{PF}_{3}$ and $\mathrm{Kr} \cdot \mathrm{PF}_{3}$ van der Waals complexes have been thoroughly investigated [1] by Fouriertransform microwave (FTMW) spectroscopy. Both of these complexes have $C_{s}$ symmetry; dipole moment data and the ${ }^{83} \mathrm{Kr}$ nuclear quadrupole coupling constants show that the rare gas atom lies over a $\mathbf{P F}_{2}$ face in both species. We have now identified the spectrum of the $\mathrm{Ne} \cdot \mathrm{PF}_{3}$ complex and find it to have $\mathrm{C}_{3 \mathrm{v}}$ symmetry with the neon lying on the threefold axis.

The microwave spectrum of $\mathrm{Ne} \cdot \mathrm{PF}_{3}$ was observed in a FTMW spectrometer [2] while investigating the $\mathrm{PF}_{3} \cdot \mathrm{H}_{2} \mathrm{O}$ system with neon as the carrier gas [3]. A number of transitions were found which exhibited first- and second-order Stark effects in the characteristic pattern of a symmetric top. These lines were visible in the absence of $\mathrm{H}_{2} \mathrm{O}$ but disappeared when argon was substituted for neon; no additional unassigned lines requiring both $\mathrm{Ne}$ and $\mathrm{PF}_{3}$ were observed. Optimum conditions for visibility were $0.1 \%$ $0.5 \% \mathrm{PF}_{3}$ in neon at a total pressure of 3.5-4 atm. The transitions were assigned to the ${ }^{20} \mathrm{Ne} \cdot \mathrm{PF}_{3}$ and ${ }^{22} \mathrm{Ne} \cdot \mathrm{PF}_{3}$ species and are listed in table 1 . Both quartic and sextic centrifugal distortion terms were needed to fit the spectrum to experimental accuracy;

1 Current address: Dow Chemical Co., Midland, MI 48667. USA. the derived rotational and centrifugal distortion constants are given in table 2 .

As $\mathrm{Ne} \cdot \mathrm{PF}_{3}$ is a symmetric top, the neon atom must lie along the symmetry axis of the $\mathrm{PF}_{3}$. Assuming that the $\mathrm{PF}_{3}$ structure [4] is unchanged upon complex. ation, the distance of the neon atom from the $\mathrm{PF}_{3}$ center-of-mass $\left(\boldsymbol{R}_{\mathrm{c} . \mathrm{m} .}\right)$ is $3.3746 \AA$ in ${ }^{20} \mathrm{Ne} \cdot \mathrm{PF}_{3}$ and $3.3704 \AA$ in ${ }^{22} \mathrm{Ne}^{2} \cdot \mathrm{PF}_{3}$. The difference presumably arises from small changes in vibrational amplitudes upon isotopic substitution. There are two choices for the neon position - over the phosphorus atom or over the $F_{3}$ face. The van der Waals radii of $\mathrm{Ne}, \mathrm{F}$, and $P$ are about $1.54,1.47$, and $1.80 \AA$, respectively [5]. For the neon over the phosphorus atom, the $\mathrm{P}-\mathrm{Ne}$ distance would be $2.875 \AA$, nearly $0.5 \AA$ too short for a van der Waals interaction. With the neon over the $\mathrm{F}_{3}$ face, the F-Ne distances are $3.385 \AA$ and the $\mathrm{P}$ Ne distance is $3.873 \AA$, all $0.4-0.5 \AA$ longer than the sum of the van der Waals radii but reasonable for a very weakly interacting system.

Millen's improved pseudodiatomic model for the van der Waals stretching motion [6] in a $C_{3 v}$ dimer gives a force constant of $f_{\mathrm{s}}=16 \pi^{2} \mu B_{0}^{3}\left(1-B_{0} / B_{\mathrm{m}}\right) /$ $D_{J}{ }^{\# 1}$, where $\mu$ is the reduced mass and $B_{\mathrm{m}}$ is the $B$ -

\#1 The symbols " $k$ " and " $f$ " are both used by various authors to indicate a harmonic force constant. Where refs. $[1,7]$ use $f_{q Q}$, we use $f_{q}$ here as in ref. [6]. Similarly, both " $\nu$ " and " $\omega$ " have been used for frequencies. 
Table 1

Assigned transitions of ${ }^{20} \mathrm{Ne} \cdot \mathrm{PF}_{3}$ and ${ }^{22} \mathrm{Ne} \cdot \mathrm{PF}_{3}$

\begin{tabular}{|c|c|c|c|c|c|c|c|}
\hline \multirow[t]{2}{*}{$J^{\prime}$} & \multirow[t]{2}{*}{$K^{\prime}$} & \multirow[t]{2}{*}{$J^{\prime \prime}$} & \multirow[t]{2}{*}{$K^{\prime \prime}$} & \multicolumn{2}{|l|}{${ }^{20} \mathrm{Ne} \cdot \mathrm{PF}_{3}$} & \multicolumn{2}{|l|}{${ }^{22} \mathrm{Ne} \cdot \mathrm{PF}_{3}$} \\
\hline & & & & $\nu_{\text {obs }}(\mathrm{MHz})^{\mathrm{a})}$ & $\nu_{\mathrm{obs}}-\nu_{\mathrm{calc}}$ & $\nu_{\text {obs }}(\mathrm{MHz})^{\text {a) }}$ & $\nu_{\text {sbs }}-v_{\text {calc }}$ \\
\hline 2 & 0 & 1 & 0 & 8081.284 & -0.0004 & 7643.0665 & 0.0004 \\
\hline 2 & 1 & 1 & 1 & 8079.353 & 0.0004 & 7641.3235 & -0.0004 \\
\hline 3 & 0 & 2 & 0 & 12119.494 & 0.0016 & 11462.364 & 0.0011 \\
\hline 3 & 1 & 2 & l & 12116.579 & -0.0020 & 11459.735 & -0.0016 \\
\hline 3 & 2 & 2 & 2 & 12107.923 & 0.0004 & 11451.925 & 0.0005 \\
\hline 4 & 0 & 3 & 0 & 16154.769 & -0.0001 & 15278.967 & 0.0003 \\
\hline 4 & 1 & 3 & 1 & 16150.861 & 0.0001 & 15275.440 & -0.0006 \\
\hline 4 & 2 & 3 & 2 & 16139.239 & 0.0003 & 15264.952 & 0.0004 \\
\hline 4 & 3 & 3 & 3 & 16120.206 & -0.0001 & 15247.768 & -0.0001 \\
\hline
\end{tabular}

a) Experimental uncertainty is $\pm 0.001-0.002 \mathrm{MHz}$.

Table 2

Rotational and distortion constants of ${ }^{20} \mathrm{Ne} \cdot \mathrm{PF}_{3}$ and ${ }^{22} \mathrm{Ne} \cdot \mathrm{PF}_{3}$

\begin{tabular}{lcc}
\hline & ${ }^{20} \mathrm{Ne} \cdot \mathrm{PF}_{3}$ & ${ }^{22} \mathrm{Ne} \mathrm{PF}_{3}$ \\
\hline$B_{0}(\mathrm{MHz})$ & $2020.64507(70)^{\text {a) }}$ & $1911.06418(58)$ \\
$D_{J}(\mathrm{kHz})$ & $40.464(75)$ & $37.176(62)$ \\
$D_{J K}(\mathrm{kHz})$ & $482.13(19)$ & $434.76(16)$ \\
$H_{J}(\mathrm{~Hz})$ & $-5.2(23)$ & $-4.7(19)$ \\
$H_{J K}(\mathrm{~Hz})$ & $-231.9(74)$ & $-216.9(61)$ \\
$H_{K S}(\mathrm{~Hz})$ & $1056.3(115)$ & $931.7(95)$ \\
\hline
\end{tabular}

a) Uncertainties are $1 \sigma$ in the last digit.

rotational constant of free $\mathrm{PF}_{3}(7819.99 \mathrm{MHz}$ [8]). Assuming a Lennard-Jones $6-12$ potential, the binding energy $\epsilon=\left(f_{\mathrm{s}} r_{\mathrm{e}}^{2} / 72\right) \approx\left(f_{\mathrm{s}} R_{\mathrm{cm} . \mathrm{m}}^{2} / 72\right)$. The derived structural and vibrational parameters are listed in table 3 along with corresponding values for $\mathrm{Ar} \cdot \mathrm{PF}_{3}$ and $\mathrm{Kr} \cdot \mathrm{PF}_{3}$ for comparison. Since the zero-point energy $\frac{1}{2} \omega_{\mathrm{s}}$ is nearly $15 \%$ of the Lennard-Jones binding energy, it is likely that anharmonic effects will be significant, explaining the difference in force constants between the ${ }^{20} \mathrm{Ne}$ and ${ }^{22} \mathrm{Ne}$ isotopic species.

A more complete description of the van der Waals vibrations may be obtained from a normal mode analysis, including the vibrational motion and force constants of the $\mathrm{PF}_{3}$, as described recently for $\mathrm{Ar} \cdot \mathrm{PF}_{3}$ and $\mathrm{Kr} \cdot \mathrm{PF}_{3}[1]$. This uses both $D_{J}\left(=-\frac{1}{4} \hbar^{4} \tau_{x x x x x}\right)$ and $D_{J K}\left(=-2 D_{J}-\frac{1}{2} \hbar^{4}\left[\tau_{x x z z}+2 \tau_{x z x z}\right]\right)$ and includes both the stretching and the doubly degenerate van der Waals bending modes; the off-diagonal force constants between these are zero by symmetry. The symmetry coordinate for the stretch is taken as
$\Delta S_{1}=\Delta R_{\text {Ne-P }}$ while for the bending these are defined as $\Delta S_{2}=\left(2 \theta_{1}-\theta_{2}-\theta_{3}\right) / \sqrt{6}$ and $\Delta S_{3}=\left(\theta_{2}-\theta_{3}\right) /$ $\sqrt{2}$, where $\theta_{1}$ is the $\mathrm{Ne}-\mathrm{P}-\mathrm{F}_{1}$ angle, etc. The results of this analysis are also listed in table 3 . The stretching force constant and frequency are nearly identical to their pseudodiatomic values, verifying the separability of the van der Waals modes from the higher frequency $\mathrm{PF}_{3}$ motions. Notable is the very small bending force constant and low bending frequency. The differences between the isotopic species again arise from the assumptions of a harmonic potential and small vibrational amplitudes; the rms stretching amplitude $\Delta R_{\text {rms }}=\left\langle\Delta R^{2}\right\rangle^{1 / 2}$ is $0.20 \AA$, while the bending amplitude is $8^{\circ}$. The difference between the effective $\left(R_{c . m}\right)$ and average $\left(R_{\text {c.m. }}^{*}\right) \mathrm{Ne}-\mathrm{PF}_{3}$ distances is $0.01 \AA$, somewhat larger than the $0.005-$ $0.006 \AA$ seen in the argon and krypton complexes.

The dipole moment was determined by measuring the second-order Stark effects of the $2_{0} \leftarrow 1_{0}, M_{J}=0$ and 1 , and the $2_{1} \leftarrow 1_{1}, M_{J}=0$ lines of ${ }^{20} \mathrm{Ne} \cdot \mathrm{PF}_{3}$ at electric field values between 0 and $600 \mathrm{~V} \mathrm{~cm}^{-1}$. The $2_{0,2} \leftarrow 1_{1,1}$ line $(12256.5835 \mathrm{MHz})$ of a trace of added $\mathrm{SO}_{2}$ was used as a field calibration standard $\left(\mu\left(\mathrm{SO}_{2}\right)=1.63305 \mathrm{D}[10]\right)$. The Stark shifts of the $\mathrm{SO}_{2}$ and dimer lines were measured sequentially at each field value. Fitting the three $M_{J}$ components independently gave essentially identical values for the dipole moment; averaging the three gives $\mu\left(\mathrm{Ne} \cdot \mathrm{PF}_{3}\right)=1.0125(10) \mathrm{D}$. This is some $0.016 \mathrm{D}$ smaller than in free $\mathrm{PF}_{3}[1]$. This small decrease may arise from an induced dipole moment in the neon atom of $0.016 \mathrm{D}$ antiparallel to the $\mathrm{PF}_{3}$ dipole mo- 
Table 3

Structural and vibrational parameters of ${ }^{20} \mathrm{Ne} \cdot \mathrm{PF}_{3}$ and ${ }^{22} \mathrm{Ne} \cdot \mathrm{PF}_{3}$

\begin{tabular}{|c|c|c|c|c|}
\hline & ${ }^{20} \mathrm{Ne} \cdot \mathrm{PF}_{3}$ & ${ }^{22} \mathrm{Ne}-\mathrm{PF}_{3}$ & $\mathrm{Ar} \cdot \mathrm{PF}_{3}{ }^{\mathrm{a})}$ & $\mathrm{Kr} \cdot \mathrm{PF}_{3}{ }^{\mathrm{a}}$ \\
\hline $\begin{array}{l}R_{\text {e.m. }}(\AA) \\
\theta_{0}{ }^{b}(\mathrm{deg})\end{array}$ & $\begin{array}{l}3.3746 \\
180.0\end{array}$ & $\begin{array}{l}3.3704 \\
180.0\end{array}$ & $\begin{array}{c}3.9533 \\
70.30\end{array}$ & $\begin{array}{l}4.0722 \\
68.25\end{array}$ \\
\hline $\begin{array}{l}f_{\mathrm{s}}{ }^{c)}\left(\operatorname{mdyn} \AA^{-1}\right) \\
\omega_{\mathrm{s}}{ }^{c)}\left(\mathrm{cm}^{-1}\right) \\
\epsilon\left(\mathrm{cm}^{-1}\right)\end{array}$ & $\begin{array}{l}0.00646 \\
25.9 \\
102.2\end{array}$ & $\begin{array}{l}0.00654 \\
25.1 \\
103.3\end{array}$ & $\begin{array}{l}0.01647 \\
31.9 \\
186.6\end{array}$ & $\begin{array}{l}0.01913 \\
27.5 \\
225.9\end{array}$ \\
\hline $\begin{array}{l}f_{R}^{\text {d) }}\left(\operatorname{mdyn} \AA^{-1}\right) \\
f_{\theta} \text { d) }(\operatorname{mdyn} \AA) \\
f_{\theta}^{\text {d) }}(\operatorname{mdyn} \AA)\end{array}$ & $\begin{array}{l}0.00647 \\
0.00464 \\
-\end{array}$ & $\begin{array}{l}0.00655 \\
0.00477 \\
-\end{array}$ & $\begin{array}{l}0.01649 \\
0.01090 \\
0.01615\end{array}$ & $\begin{array}{l}0.01933 \\
0.01537 \\
0.01797\end{array}$ \\
\hline $\begin{array}{l}\omega_{R}^{d \prime}\left(\mathrm{cm}^{-1}\right) \\
\omega_{\theta}^{d \prime}\left(\mathrm{cm}^{-1}\right) \\
\omega_{\phi}^{d)}\left(\mathrm{cm}^{-1}\right)\end{array}$ & $\begin{array}{l}26.0 \\
13.7 \\
-\end{array}$ & $\begin{array}{c}25.1 \\
13.7 \\
-\end{array}$ & $\begin{array}{l}31.8 \\
18.1 \\
19.6\end{array}$ & $\begin{array}{l}27.6 \\
20.9 \\
20.3\end{array}$ \\
\hline $\begin{array}{l}\Delta R_{\mathrm{rms}}{ }^{\mathrm{d})}(\AA) \\
\Delta \theta_{\mathrm{rms}}{ }^{\mathrm{d})}(\mathrm{deg}) \\
\Delta \varphi_{\mathrm{rms}}^{\mathrm{d})}(\mathrm{deg})\end{array}$ & $\begin{array}{l}0.200 \\
8.0 \\
-\end{array}$ & $\begin{array}{l}0.197 \\
7.9 \\
-\end{array}$ & $\begin{array}{l}0.153 \\
10.2 \\
7.1\end{array}$ & $\begin{array}{l}0.136 \\
9.7 \\
6.8\end{array}$ \\
\hline$R_{\text {c.m. }}^{*}{ }^{d)}(\AA)$ & 3.3847 & 3.3801 & 3.9595 & 4.0774 \\
\hline
\end{tabular}

a) Ref. [1].

b) $\theta_{0}$ is the $P-X-R G$ angle, where $X$ is the center of mass of the $P F_{3}$ and $R G$ is the rare-gas atom.

c) Calculated using the pscudodiatomic model of ref. [6].

d) Calculated from a normal mode analysis (see text), $R$ refers to the stretching coordinate, analogous to $f_{\text {s: }} \theta$ refers to the symmetric (in-plane) bend and $\Phi$ to the asymmetric bend $\left(\mathrm{PF}_{3}\right.$ torsion) for $\mathrm{Ar} \cdot \mathrm{PF}_{3}$ and $\mathrm{Kr} \cdot \mathrm{PF}_{3} . R_{\mathrm{c} . \mathrm{m} \text {. }}^{*}$ is the average center of mass distance [9].

ment. Alternatively, it can be explained by a vibrational averaging of the projection of $\mu\left(\mathrm{PF}_{3}\right)$ onto the $\mathrm{C}_{3}$ axis of the complex. Estimating the average bending angle as $\cos ^{-1}\left[\mu\left(\mathrm{PF}_{3} \cdot \mathrm{Ne}\right) / \mu\left(\mathrm{PF}_{3}\right)\right]$ gives $\langle\Delta \Theta\rangle$ $=10.3^{\circ}$; the good agreement with the vibrational analysis above suggests that the induced dipole moment in the neon is somewhat less than $0.016 \mathrm{D}$.

To further explore the change in the dipole moment, an ab initio calculation on free $\mathrm{PF}_{3}$, using the GAUSSIAN 86 package with a $6-31 G^{*}$ basis set [11], was used to determine the electric field at the neon site. The use of ab initio calculations on the free polar species has been successful at modeling both electric fields (i.c. dipole moments) and field gradients (via ${ }^{83} \mathrm{Kr}$ nuclear quadrupole coupling) in a number of polar-nonpolar van der Waals complexes $[1,7,12]$. $A$ map of the calculated field around $\mathrm{PF}_{3}$ is shown in fig. 1. It is interesting to note that the $\mathrm{Ar}$ and $\mathrm{Kr}$ sites both exhibit a large positive electrostatic potential while the Ne site has a negative potential. The calculated field at the neon site combined with the polarizability of neon $\left(0.364 \AA^{3}[13]\right)$ predicts an induced dipole moment of only $0.0003 \mathrm{D}$, suggesting that vibrational averaging rather than polarization accounts for most of the decrease in the dipole moment of the complex.

The small induced moment and negative site polarity both suggest that electrostatic interactions contribute little to the binding in $\mathrm{Ne} \cdot \mathrm{PF}_{3}$. An upper limit to the polarization contribution to the binding energy can be set by assuming that all of the change in the dipole moment arises from polarization of the rare-gas atom, and that higher-order induced moment terms are small compared to the induced dipole contribution. The interaction energy is then $\Delta E=-\mu_{\text {ind }}^{2} / 2 \alpha$ [14], where $\mu_{\text {ind }}$ is the induced dipole moment and $\alpha$ is the polarizability in the raregas atom. For the neon, argon and krypton complexes this gives stabilization energies of $-1.6,-16.6$ and $-17.7 \mathrm{~cm}^{-1}$, respectively, confirming the small electrostatic interaction in $\mathrm{Ne} \cdot \mathrm{PF}_{\mathbf{3}}$.

To further explore the interactions in this system, a series of HF/SCF ab initio calculations was made on the $\mathrm{Ne} \cdot \mathrm{PF}_{3}$ supermolecule using the $6-31+\mathrm{G}^{*}$ basis set (the diffuse exponent for $\mathrm{Ne}$ was set to 0.125 ), with some smaller basis sets also used. Due 


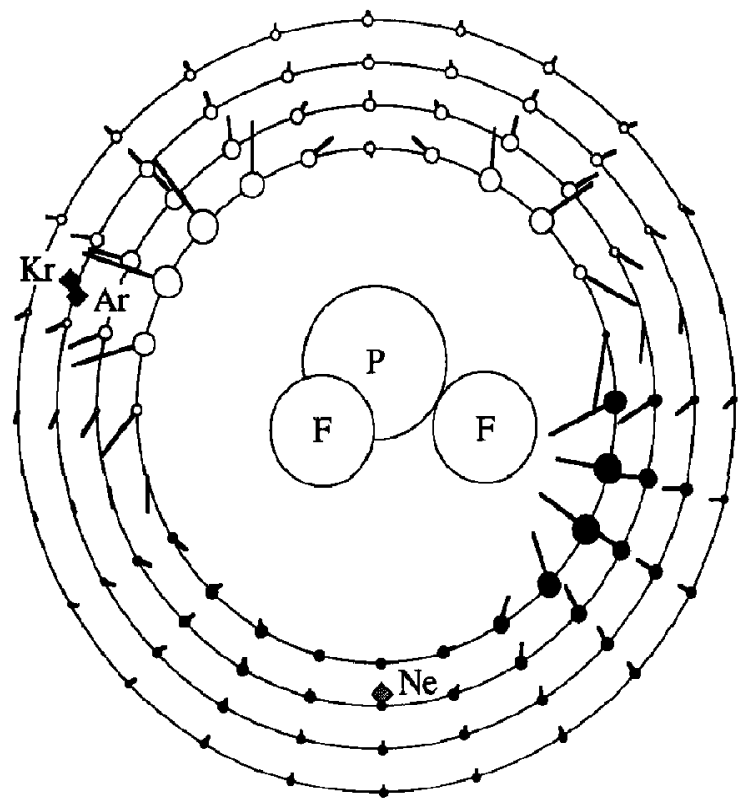

Fig. 1. A map of the electric field around $\mathrm{PF}_{3}$, Open circles indicate a positive electrostatic potential and filled circles a negative potential; the magnitude is indicated by their size. The lines indicate the direction and magnitude of the field. Values are calculated at $R_{\text {c.m. }}=3.0,3.5,4.0$ and $4.5 \AA$ with $15^{\circ}$ increments in $\theta$. The locations of $\mathrm{Ne}, \mathrm{Ar}$ and $\mathrm{Kr}$ in their respective complexes are indicated.

to CPU time constraints, MP and CI calculations were not feasible. The potential energy curve calculated for a $\mathrm{C}_{3 v}$ geometry with the neon over the $\mathrm{F}_{3}$ face is shown in fig. 2. The minimum energy structure $\left(\Delta E \approx-20 \mathrm{~cm}^{-1}\right)$ was at $R_{\text {c.m. }}=3.5-3.6 \dot{\mathrm{A}}$; no minimum was found for neon over the $P$ atom. Given the level of the calculations (SCF with diffuse orbitals) it seems reasonable to believe that the electrostatic interactions in the complex will be fairly well modeled but the dispersion interactions will not; the very small binding energy found is consistent with this interpretation. It is interesting in this context that we find a second minimum of nearly equal energy for a structure with the neon over the $\mathrm{PF}_{2}$ face, roughly at the site predicted by analogy to $\mathrm{Ar} \cdot \mathrm{PF}_{3}$ and $\mathrm{Kr} \cdot \mathrm{PF}_{3}$.

There has been some debate in the literature over the utility of the counterpoise correction to eliminate basis set superposition error (BSSE) [15]; the large polarization + diffuse orbital basis set was used in the hope of minimizing BSSE without the computa-

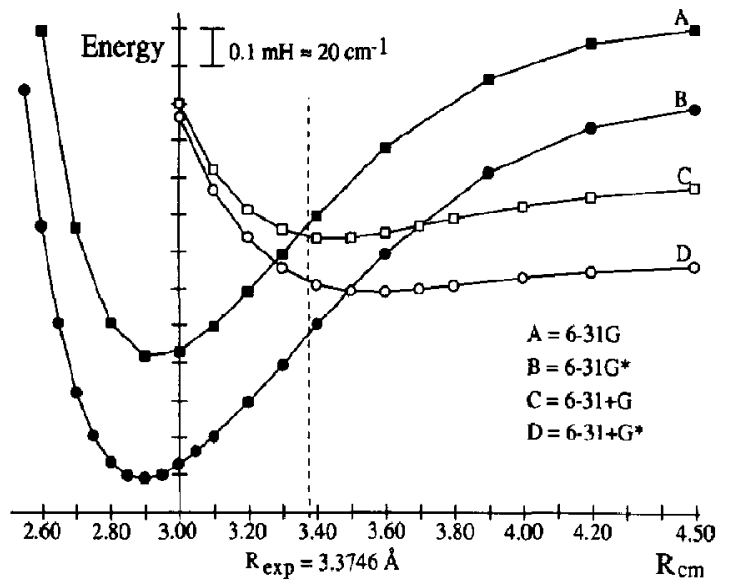

Fig. 2. The ab initio energy versus $R_{\text {c.m. }}$ for $\mathrm{Ne} \cdot \mathrm{PF}_{3}$ in a $\mathrm{C}_{3 v}$ geometry, calculated with the $6-31+\mathrm{G}^{*}$ basis set. The total dimer energy at $R_{\text {c.m. }}=4.50 \AA$ is -767.62911152 hartree.

tional burden of counterpoise calculations. Trial counterpoise calculations were done for the $\mathrm{C}_{3 \mathrm{v}}$ geometries of $\mathrm{PF}_{3} \cdot \mathrm{Ne}$; somewhat surprisingly, no minimum in the potential energy could be found.

As the electrostatic contribution to the binding energy in $\mathrm{Ne} \cdot \mathrm{PF}_{3}$ is small it is clear that the dispersion interactions dominate here, necessitating a more extensive theoretical treatment including electron correlation for an accurate description. Nevertheless it is attractive to rationalize the structures of the raregas $\mathrm{PF}_{3}$ complexes as a balance of polarization and dispersion interactions. In the argon and krypton complexes the larger size and higher polarizabilities force the atom into a site of large field, positive potential, and contact with three of the partner's atoms. For $\mathrm{Ne}-\mathrm{PF}_{3}$ the neon atom nestles into the open face of the $\mathrm{PF}_{3}$ pyramid allowing a dispersion interaction with all four partner atoms.

This work has been supported by grants from the National Science Foundation, Washington DC. We appreciate several helpful comments made by the referee.

\section{References}

[1] A. Taleb-Bendiab, M.S. LaBarge, L.L. Lohr, R.C. Taylor, K.W. Hillig II, R.L. Kuczkowski and R.K. Bohn, J. Chem. Phys. 90 (1989) 6949. 
[2] K.W. Hillig II, J. Matos, A. Scioly and R.L. Kuczkowski, Chem. Phys. Letters 133 (1987) 359.

[3] K.W. Hillig II, M.S. LaBarge, R.K. Bohn and R.L. Kuczkowski, unpublished work.

[4] M.D. Harmony, Y.W. Laurie, R.L. Kuczkowski, R.H. Schwendeman, D.A. Ramsey, F.J. Lovas, W.J. Lafferty and A.G. Maki, J. Phys. Chem. Ref. Data 8 (1979) 619.

[5] A. Bondi, J. Phys. Chem. 68 (1964) 441.

[6] D.J. Millen, Can. J. Chem. 63 (1985) 1477.

[7] J.J. Oh, K.W. Hillig I, R.L. Kuczkowski and R.K. Bohn, J. Phys. Chem, in press.

[8] A.M. Mirri, F. Scappini and F.G. Favero, Spectrochim. Acta 21 (1965) 965.

[9] D.R. Herschbach and V.W. Laurie, J. Chem. Phys. 37 (1962) 1668.

[10] D. Patel, D. Margolese and T.R. Dyke, J. Chem. Phys. 70 (1979) 2740.

[11] M.J. Frisch, J.S. Binkley, H.B. Schlegel, K. Raghavachari, C.F. Melius, R.L. Martin, J.J.P. Stewart, F.W. Bobrowicz, C.M. Rohlfing, L.R. Kahn, D.J. DeFrees, R. Seeger, R.A.
Whiteside, D.J. Fox, E.M. Fluder and J.A. Pople, GAUSSIAN 86, Carnegie-Mellon Quantum Chemistry Publishing Unit, Pittsburgh, PA (1986).

[12] A.M. Andrews, A. Taleb-Bendiab, M.S. LaBarge, K.W. Hillig II and R.L. Kuczkowski, J. Chem. Phys., submitted for publication; unpublished results.

[13] R.H. Orcutt and R.H. Cole, J. Chem. Phys. 46 (1967) 697.

[14] E.A. Moelwyn-Hughes, Physical chemistry, 2nd Ed. (Pergamon Press, New York, 1961) p. 310.

[15] S.F. Boys and F. Bernardi, Mol. Phys. 19 (1970) 553; D.W. Schwenke and D.G. Truhlar, J. Chem. Phys. 82 (1985) 2418;

S.K. Loushin, S.-Y, Liu and C.D. Dykstra, J. Chem. Phys. 82 (1985) 2720;

M.M. Szczęsniak and S. Scheiner, J. Chem. Phys. 84 (1986) 6328. 